\title{
Simplified Transfer Matrix Approach in the Two-Dimensional Ising Model with Various Boundary Conditions
}

\author{
Boris Kastening \\ Institut für Theoretische Physik \\ Freie Universität Berlin \\ Arnimallee 14 \\ D-14195 Berlin \\ Germany \\ (Dated: 24 May 2002)
}

\begin{abstract}
A recent simplified transfer matrix solution of the two-dimensional Ising model on a square lattice with periodic boundary conditions is generalized to periodic-antiperiodic, antiperiodic-periodic and antiperiodic-antiperiodic boundary conditions. It is suggested to employ linear combinations of the resulting partition functions to investigate finite-size scaling. An exact relation of such a combination to the partition function corresponding to Brascamp-Kunz boundary conditions is found.
\end{abstract}

PACS numbers: 05.50.+q, 68.35.Rh, 05.70.Ce, 64.60.Cn

Recently, a solution of the two-dimensional Ising Model on a square lattice with periodic boundary conditions was presented [1], which simplifies Kaufman's version [2] of Onsager's solution [3] without requiring sophisticated mathematics. Here we point out how the method easily generalizes also to the cases of periodic-antiperiodic, antiperiodic-periodic and antiperiodic-antiperiodic boundary conditions, which were recently solved using a Grassmann path integral approach [4. The motivation for varying the boundary conditions originates in the investigation of finitesize scaling (FSS) [5, 6, 7]. The analytic properties of FSS are of interest when trying to describe critical properties of models whose exact solution is unknown. For the analysis of FSS in solvable models it is very helpful if the exact partition function can be written as a product rather than as a sum [6, 7] as is the case with Brascamp-Kunz (BK) boundary conditions [8]. Here we exhibit linear combinations of partition functions with varying boundary conditions that have this property and may be used for such an analysis. For one of these linear combinations we find an exact relation to the partition function for BK boundary conditions.

We follow the notation in [1]. Define the energy $E$ of the two-dimensional Ising model on a square lattice with $m \times n$ sites by

$$
\begin{aligned}
-\beta E= & a \sum_{\nu=1}^{n}\left(\sigma_{a} s_{m \nu} s_{1 \nu}+\sum_{\mu=1}^{m-1} s_{\mu \nu} s_{\mu+1, \nu}\right) \\
& +b \sum_{\mu=1}^{m}\left(\sigma_{b} s_{\mu n} s_{\mu 1}+\sum_{\nu=1}^{n-1} s_{\mu \nu} s_{\mu, \nu+1}\right),
\end{aligned}
$$

where we assume $a, b>0$ as in [1] and where the spin variables $s_{\mu \nu}$ can assume the values $\pm 1 . \quad\left(\sigma_{a}, \sigma_{b}\right)=$ $(1,1)$ corresponds to periodic boundary conditions in

*Email address: ka@physik.fu-berlin.de both directions (pp), as investigated in [1, 2, 3]. $\left(\sigma_{a}, \sigma_{b}\right)=(1,-1),(-1,1)$ and $(-1,-1)$ correspond to periodic-antiperiodic (pa), antiperiodic-periodic (ap) and antiperiodic-antiperiodic (aa) boundary conditions, respectively. The partition function is defined by

$$
Z_{\alpha \beta}^{(m, n)}(a, b)=\sum_{s_{\mu \nu}= \pm 1} \exp (-\beta E)
$$

where $\alpha \beta$ may be pp, pa, ap or aa. After defining $2^{n} \times 2^{n}$ matrices $\mathrm{X}_{\nu}$ (and analogously $\mathrm{Y}_{\nu}$ and $\mathrm{Z}_{\nu}$ ) by

$$
\mathrm{X}_{\nu}=\underbrace{\mathbf{1} \otimes \cdots \otimes \mathbf{1}}_{\nu-1} \otimes \sigma_{x} \otimes \underbrace{\mathbf{1} \otimes \cdots \otimes \mathbf{1}}_{n-\nu}
$$

where $\sigma_{x}$ is a Pauli matrix and $\mathbf{1}$ is the $2 \times 2$ unit matrix, we may write the partition function as

$$
Z_{\alpha \beta}^{(m, n)}(a, b)=[2 \sinh (2 a)]^{m n / 2} \operatorname{Tr}\left(\mathrm{QV}^{m}\right)
$$

where $\mathrm{Q}=\mathbf{1}$ (now the $2^{n} \times 2^{n}$ unit matrix) and $\mathrm{Q}=$ $\mathrm{U}_{\mathrm{X}} \equiv \mathrm{X}_{1} \mathrm{X}_{2} \ldots \mathrm{X}_{n}$ for periodic and antiperiodic boundary conditions in the $a$ direction, respectively, and where

$$
\mathrm{V}=\mathrm{V}_{a / 2} \mathrm{~V}_{b} \mathrm{~V}_{a / 2}
$$

with

$$
\mathrm{V}_{a / 2}=\prod_{\nu=1}^{n} \exp \left(\bar{a} \mathrm{X}_{\nu} / 2\right),
$$

where $\bar{a}$ is defined by $\sinh 2 a \sinh 2 \bar{a}=1$ as in [1], and

$$
\mathrm{V}_{b}=\exp \left(\sigma_{b} b \mathrm{Z}_{n} \mathrm{Z}_{1}\right) \prod_{\nu=1}^{n-1} \exp \left(b \mathrm{Z}_{\nu} \mathrm{Z}_{\nu+1}\right)
$$

with $\sigma_{b}=1$ and $\sigma_{b}=-1$ for periodic and antiperiodic boundary conditions in the $b$ direction, respectively. With the same set of matrices

$$
\mathrm{J}_{\alpha \beta}^{ \pm}=\mathrm{P}^{ \pm} \mathrm{J}_{\alpha \beta}, \quad \mathrm{P}^{ \pm} \equiv \frac{1}{2}\left(\mathbf{1} \pm \mathrm{U}_{\mathrm{X}}\right)
$$


as in [1], where

$$
\begin{aligned}
\mathrm{J}_{\alpha \beta} & =-\frac{i}{4}\left[\Gamma_{\alpha}, \Gamma_{\beta}\right], \\
\Gamma_{2 \nu-1} & =\mathrm{X}_{1} \cdots \mathrm{X}_{\nu-1} \mathrm{Z}_{\nu}, \\
\Gamma_{2 \nu} & =\mathrm{X}_{1} \cdots \mathrm{X}_{\nu-1} \mathrm{Y}_{\nu},
\end{aligned}
$$

the partition function may be rewritten as

$$
Z_{\alpha \beta}^{(m, n)}(a, b)=[2 \sinh (2 a)]^{m n / 2} \operatorname{Tr}\left(\mathrm{Q}_{\alpha} \mathrm{V}_{\beta}^{m}\right)
$$

with

$$
\begin{gathered}
\mathrm{Q}_{\left\{\begin{array}{c}
\mathrm{p}\} \\
\mathrm{a}
\end{array}\right.}=\mathrm{P}_{+} \pm \mathrm{P}_{-}, \\
\mathrm{V}_{\beta}=\mathrm{V}_{\beta}^{+} \mathrm{V}_{\beta}^{-}=\mathrm{V}_{\beta}^{-} \mathrm{V}_{\beta}^{+}, \\
\mathrm{V}_{\beta}^{ \pm}=\mathrm{V}_{a / 2}^{ \pm} \mathrm{V}_{b \beta}^{ \pm} \mathrm{V}_{a / 2}^{ \pm}, \\
\mathrm{V}_{a / 2}^{ \pm}=\prod_{\nu=1}^{n} \exp \left(\bar{a} J_{2 \nu, 2 \nu-1}^{ \pm}\right), \\
\mathrm{V}_{b \mathrm{p}}^{ \pm}=\exp \left(\mp 2 b \mathrm{~J}_{1,2 n}^{ \pm}\right) \prod_{\nu=1}^{n-1} \exp \left(2 b \mathrm{~J}_{2 \nu+1,2 \nu}^{ \pm}\right), \\
\mathrm{V}_{b \mathrm{a}}^{ \pm}=\exp \left( \pm 2 b \mathrm{~J}_{1,2 n}^{ \pm}\right) \prod_{\nu=1}^{n-1} \exp \left(2 b \mathrm{~J}_{2 \nu+1,2 \nu}^{ \pm}\right)
\end{gathered}
$$

From here on it is straightforward to follow the arguments given in [1] to compute the partition function in terms of the $\gamma_{k}$ defined by

$$
\cosh \gamma_{k}=\cosh 2 \bar{a} \cosh 2 b-\cos \frac{\pi k}{n} \sinh 2 \bar{a} \sinh 2 b
$$

with the understanding that $\gamma_{k}>0$ for $1 \leq k \leq n$ and with $\gamma_{0}=2(\bar{a}-b)$. It turns out that antiperiodic boundary conditions in the $b$ direction lead to an exchange of $\gamma_{k}$ with even and odd indices, while antiperiodic boundary conditions in the $a$ direction lead to a sign change of half of the terms in the partition function, see (32) and (33) below.

Define

$$
\begin{aligned}
& \Sigma_{\mathrm{ee}}^{(m, n)}(a, b)=\sum_{\mathrm{e}} \exp \left(\frac{m}{2} \sum_{\nu=1}^{n}( \pm) \gamma_{2 \nu-2}\right), \\
& \Sigma_{\mathrm{eo}}^{(m, n)}(a, b)=\sum_{\mathrm{e}} \exp \left(\frac{m}{2} \sum_{\nu=1}^{n}( \pm) \gamma_{2 \nu-1}\right), \\
& \Sigma_{\mathrm{oe}}^{(m, n)}(a, b)=\sum_{\mathrm{o}} \exp \left(\frac{m}{2} \sum_{\nu=1}^{n}( \pm) \gamma_{2 \nu-2}\right), \\
& \Sigma_{\mathrm{oO}}^{(m, n)}(a, b)=\sum_{\mathrm{o}} \exp \left(\frac{m}{2} \sum_{\nu=1}^{n}( \pm) \gamma_{2 \nu-1}\right),
\end{aligned}
$$

where the first index in $\Sigma_{x y}$ and the index under the summation sign refer to all combinations in the sum in the exponent that have an even/odd number of minus signs and the second index of $\Sigma_{\mathrm{xy}}$ refers to even/odd indices of the $\gamma_{k}$. Define also

$$
\begin{aligned}
& C_{\mathrm{o}}^{(m, n)}(a, b)=[2 \sinh (2 a)]^{m n / 2} \prod_{k=1}^{n}\left[2 \cosh \left(\frac{m}{2} \gamma_{2 k-1}\right)\right], \\
& S_{\mathrm{o}}^{(m, n)}(a, b)=[2 \sinh (2 a)]^{m n / 2} \prod_{k=1}^{n}\left[2 \sinh \left(\frac{m}{2} \gamma_{2 k-1}\right)\right], \\
& C_{\mathrm{e}}^{(m, n)}(a, b)=[2 \sinh (2 a)]^{m n / 2} \prod_{k=1}^{n}\left[2 \cosh \left(\frac{m}{2} \gamma_{2 k-2}\right)\right],
\end{aligned}
$$

$$
S_{\mathrm{e}}^{(m, n)}(a, b)=[2 \sinh (2 a)]^{m n / 2} \prod_{k=1}^{n}\left[2 \sinh \left(\frac{m}{2} \gamma_{2 k-2}\right)\right],
$$

and note that

$$
\begin{aligned}
& S_{\mathrm{o}}^{(2 m, n)}(a, b)=S_{\mathrm{o}}^{(m, n)}(a, b) C_{\mathrm{o}}^{(m, n)}(a, b), \\
& S_{\mathrm{e}}^{(2 m, n)}(a, b)=S_{\mathrm{e}}^{(m, n)}(a, b) C_{\mathrm{e}}^{(m, n)}(a, b) .
\end{aligned}
$$

Then, omitting upper indices $(m, n)$ and arguments $(a, b)$ for brevity, we have for the various cases

$$
\begin{aligned}
& Z_{\mathrm{pp}}=[2 \sinh (2 a)]^{m n / 2}\left(\Sigma_{\mathrm{eo}}+\Sigma_{\mathrm{oe}}\right)=\frac{1}{2}\left(C_{\mathrm{o}}+S_{\mathrm{o}}+C_{\mathrm{e}}-S_{\mathrm{e}}\right), \\
& Z_{\mathrm{pa}}=[2 \sinh (2 a)]^{m n / 2}\left(\Sigma_{\mathrm{ee}}+\Sigma_{\mathrm{oo}}\right)=\frac{1}{2}\left(C_{\mathrm{o}}-S_{\mathrm{o}}+C_{\mathrm{e}}+S_{\mathrm{e}}\right), \\
& Z_{\mathrm{ap}}=[2 \sinh (2 a)]^{m n / 2}\left(\Sigma_{\mathrm{eo}}-\Sigma_{\mathrm{oe}}\right)=\frac{1}{2}\left(C_{\mathrm{o}}+S_{\mathrm{o}}-C_{\mathrm{e}}+S_{\mathrm{e}}\right), \\
& Z_{\mathrm{aa}}=[2 \sinh (2 a)]^{m n / 2}\left(\Sigma_{\mathrm{ee}}-\Sigma_{\mathrm{oo}}\right)=\frac{1}{2}\left(-C_{\mathrm{o}}+S_{\mathrm{o}}+C_{\mathrm{e}}+S_{\mathrm{e}}\right) .
\end{aligned}
$$


The result for $Z_{\mathrm{pp}}$ is the one found in [1] and, observing the different sign convention for $\gamma_{0}$, in 2 .

In [6, 7]. FSS was investigated with BK boundary conditions [8] so that the partition function has a product structure which facilitates the subsequent analysis of the approach to the critical point in the thermodynamic limit. The partition functions above do not have such a product structure in contrast to their linear combinations $C_{\mathrm{o}}, S_{\mathrm{o}}, C_{\mathrm{e}}$ and $S_{\mathrm{e}}$. Except for $S_{\mathrm{e}}$, these combinations are positive for any temperature and may be viewed as partition functions belonging to models with possibly nonlocal boundary conditions. Even though these boundary conditions may in general not be particularly physical, the quantities $C_{\mathrm{o}}, S_{\mathrm{o}}$ and $C_{\mathrm{e}}$ may be used as a mathematical tool to investigate the approach to criticality in the same way as the partition function of the model with BK boundary conditions.

$S_{\mathrm{e}}$ does not qualify for such an approach. Since the pp boundary conditions cause a lower ground state energy than the other three boundary conditions considered here, $S_{\mathrm{e}}$ becomes negative for sufficiently low temperatures since $Z_{\mathrm{pp}}$ enters with a minus sign. $S_{\mathrm{e}}$ has another interesting property, though: Due to the sign change of $\gamma_{0}$ at the critical temperature of the thermodynamic limit, (i.e., where $\bar{a}=b$ ), $S_{\mathrm{e}}$ changes its sign at the critical temperature for any size $m \times n$ of the square lattice.

The quantities (24)-(27) are the same as in [5], even though they have a different appearance. In our notation, the expressions in [5] read

$$
\begin{aligned}
C_{\mathrm{o}}^{(m, n)}(a, b)= & 2^{m n} \prod_{p=0}^{m-1} \prod_{q=0}^{n-1}\left[\cosh (2 a) \cosh (2 b)-\cos \frac{(2 p+1) \pi}{m} \sinh (2 a)-\cos \frac{(2 q+1) \pi}{n} \sinh (2 b)\right]^{1 / 2}, \\
S_{\mathrm{o}}^{(m, n)}(a, b)= & 2^{m n} \prod_{p=0}^{m-1} \prod_{q=0}^{n-1}\left[\cosh (2 a) \cosh (2 b)-\cos \frac{2 p \pi}{m} \sinh (2 a)-\cos \frac{(2 q+1) \pi}{n} \sinh (2 b)\right]^{1 / 2}, \\
C_{\mathrm{e}}^{(m, n)}(a, b)= & 2^{m n} \prod_{p=0}^{m-1} \prod_{q=0}^{n-1}\left[\cosh (2 a) \cosh (2 b)-\cos \frac{(2 p+1) \pi}{m} \sinh (2 a)-\cos \frac{2 q \pi}{n} \sinh (2 b)\right]^{1 / 2}, \\
S_{\mathrm{e}}^{(m, n)}(a, b)= & \operatorname{sgn}(1-\sinh (2 a) \sinh (2 b)) \times \\
& 2^{m n} \prod_{p=0}^{m-1} \prod_{q=0}^{n-1}\left[\cosh (2 a) \cosh (2 b)-\cos \frac{2 p \pi}{m} \sinh (2 a)-\cos \frac{2 q \pi}{n} \sinh (2 b)\right]^{1 / 2} .
\end{aligned}
$$

While these expressions involve a double product instead of just a single product as in (24)-(27), they have the advantage that $C_{o}$ and $S_{e}$ are symmetric under the exchange $(a, m) \leftrightarrow(b, n)$ and that the only asymmetry of $C_{e}$ and $S_{o}$ under this exchange originates in the boundary conditions and not in the mathematical treatment.

While $\mathrm{Wu}$ et al. [4 use the Grassmannian integral approach to map the problem onto a free fermion system, the method employed in this work is close in spirit to Kaufman's solution [2], but avoiding some complications encountered there, as detailed in [1]. While the Grassmannian method may be viewed as more elegant, our method is accessible at a less sophisticated mathematical level. It is also interesting to note the very different appearance of the solutions (24)-(27) and (34)-(37). It would be interesting to see if our method can be generalized to cases that so far have been solved only using the Grassmannian integral approach, such as various boundary conditions on plane-triangular and honeycomb lattices [5], or other.

Brascamp and Kunz [8] gave a solution for a twodimensional Ising model on a square lattice with $(m+2) \times$ $n$ sites with periodic boundary conditions in the $b$ direction and fixed boundary conditions in the $a$ direction. If $k$ enumerates the sites in the $a$ direction $(0 \leq k \leq m+1)$, then the spins at $k=0$ are fixed at +1 , while the spins at $k=m+1$ are fixed to alternate between -1 and +1 , so $n$ must be even. The resulting partition function was determined to be 8

$$
Z_{\mathrm{BK}}^{(m, n)}(a, b)=2^{m n} \prod_{p=1}^{m} \prod_{q=1}^{n / 2}\left[\cosh (2 a) \cosh (2 b)-\cos \frac{p \pi}{m+1} \sinh (2 a)-\cos \frac{(2 q-1) \pi}{n} \sinh (2 b)\right]
$$

Using (28) and (34)-(36), we can establish the connection

$$
=C_{\mathrm{e}}^{(n, 2)}(b, a) Z_{\mathrm{BK}}^{(m, n)}(a, b)^{2},
$$

$$
S_{\mathrm{o}}^{(2(m+1), n)}(a, b)=S_{\mathrm{o}}^{(2, n)}(a, b) Z_{\mathrm{BK}}^{(m, n)}(a, b)^{2}
$$


where the $S_{\text {o }}$ may be factorized according to 28) and $C_{\mathrm{e}}^{(n, 2)}(b, a)$ most easily evaluated using (26).

Eq. (39) generalizes Eq. (10) in 7 to the case $a \neq b$. It would be interesting to understand (39) without having to resort to the solutions of the models. Then it may also be possible to relate $C_{\mathrm{o}}, S_{\mathrm{o}}, C_{\mathrm{e}}$ and $S_{\mathrm{e}}$ to yet other physical boundary conditions.

Investigation of FSS for $C_{\mathrm{o}}, S_{\mathrm{o}}$ and $C_{\mathrm{e}}$ is beyond the scope of this paper. Let us however state one interesting result that can be read off immediately from (34). If $a=b$ and we define $z=\sinh 2 a$, the zeros $z_{p q}$ of $C_{\mathrm{o}}$ in the complex plane are given by $z_{p q}=\exp \left(i \alpha_{p q}\right)$ with

$$
\alpha_{p q}=\arccos \left[\frac{1}{2}\left(\cos \frac{(2 p+1) \pi}{m}+\cos \frac{(2 q+1) \pi}{n}\right)\right]
$$

and the zero nearest to the real axes is found for $p=q=$ 0 . For $m=n$, this zero scales as

$$
\alpha_{00}=\frac{\pi}{n}
$$

without any corrections as opposed to the case of BK boundary conditions [6, 7]. It remains to be investigated if simplifications may also be achieved for the FSS of other quantities and if simplifying combinations of partition functions can also be defined for models whose exact solution is unavailable, e.g. by the use of symmetry arguments.
[1] B. Kastening, Pedestrian Solution of the TwoDimensional Ising Model, cond-mat/0104398; Phys. Rev. E 64, 066106 (2001) cond-mat/0111380.

[2] B. Kaufman, Phys. Rev. 76, 1232 (1949).

[3] L. Onsager, Phys. Rev. 65, 117 (1944).

[4] M.-C. Wu, M.-C. Huang, Y.-P. Luo and T.-M. Liaw, J. Phys. A: Math. Gen. 32, 4897 (1999).

[5] M.-C. Wu and C.-K. Hu, J. Phys. A: Math. Gen. 35, 5189
(2002) cond-mat/0204217.

[6] W. Janke and R. Kenna, Phys. Rev. B65, 064110 (2002) cond-mat/0103332]; Nucl.Phys.Proc.Suppl. 106, 929 (2002) hep-lat/0112037.

[7] N. Sh. Izmailian, K. B. Oganesyan, and C.-K. Hu, Phys. Rev. E65, 056132 (2002) cond-mat/0202282.

[8] H.J. Brascamp and H. Kunz, J. Math. Phys. 15, 65 (1974). 